KYUNGPOOK Math. J. 52(2012), 405-412

http://dx.doi.org/10.5666/KMJ.2012.52.4.405

\title{
A Unified Theory for Certain Weak Forms of Open Sets and Their Variant Forms
}

\author{
BishwambHaR RoY* \\ Department of Mathematics, Women's Christian College, 6, Greek Church Row, \\ Kolkata 700 026, India \\ e-mail : bishwambhar_roy@yahoo.co.in \\ RITU SEN ${ }^{\dagger}$ \\ Department of Mathematics, S. A. Jaipuria College, 10, Raja Naba Krishna Street, \\ Kolkata 700 005, India \\ e-mail: ritu_sen29@yahoo.co.in
}

Abstract. The purpose of the present paper is towards working out a unified version of the study of certain weak forms of generalized open sets and their neighbouring forms, as are already available in the literature. In terms of an operation, as initiated by Á. Császár, we introduce unified definitions of $\bigwedge_{\psi}{ }^{- \text {sets, }} \bigvee_{\psi}$-sets, $g \cdot \bigwedge_{\psi}$-sets and $g \cdot \bigvee_{\psi}$-sets and derive results concerning them.

\section{Introduction}

It is observed from literature that there has been a considerable work on different relatively weak forms of generalized open sets, like $g . \wedge$-sets and $g . \bigvee$-sets in particular; several other neighbouring forms of them have also been studied in many papers. For instance, $g . \bigwedge_{s}$-sets, $g \bigwedge_{p^{- \text {sets}}} g \bigwedge_{\delta^{-}}$-sets, $g . \bigwedge_{\alpha}$-sets, $g . \wedge s p$-sets are some of the variant forms of $g$. $\wedge$-sets, that have been investigated by different researchers as separate entities. Similar observations are applicable to $g$. $\bigvee$-sets. As can be observed, all these variations can be achieved by using different types of operators like int, intcl, intcl $_{\delta}$, clint $_{\delta}$, clint, intclint, clintcl, where int and $c l$ respectively stand for interior and closure operators, and $c l_{\delta}$ denotes the $\delta$-closure operator. The concept of a generalized type of operator, called operation on the power set $\mathcal{P}(X)$ of a topological space $(X, \tau)$, was introduced in [6]. It turns out from the investigations that by judicious use of the notion of 'operation', one can give generalized definitions of $\bigwedge_{\psi}$-sets, $\bigvee_{\psi}$-sets, $g$. $\bigwedge_{\psi}$-sets and $g . \bigvee_{\psi}$-sets from which the definitions of different varied forms of generalized sets follow and as a result

\footnotetext{
* Corresponding Author.

Received March 7, 2011; accepted June 4, 2012.

2010 Mathematics Subject Classification: 54C08, 54D10, 54D15.

Key words and phrases: Operation, $\psi$-closure, $\psi$-open set, $\bigwedge_{\psi}$-sets, $g \cdot \bigwedge_{\psi}$-sets.

$\dagger$ The author acknowledges the financial support from UGC, New Delhi.
} 
we can obtain their properties and results concerning them. A similar attempts towards such a generalization under the terminology " $\bigwedge_{\mu}$-sets and $\bigvee_{\mu}$-sets" was undertaken by Ekici and Roy in [8].

\section{2. $\bigwedge_{\psi}$-sets and $\bigvee_{\psi}$-sets}

We now begin by recalling a few definitions and observe that many of the existing relevant definitions considered in various papers turn out to be special cases of the ones given below.

Definition 2.1([6]). Let $(X, \tau)$ be a topological space. A mapping $\psi: \mathcal{P}(X) \rightarrow$ $\mathcal{P}(X)$ is called an operation on $\mathcal{P}(X)$, where $\mathcal{P}(X)$ denotes as usual the power set of $X$, if for each $A \in \mathcal{P}(X) \backslash\{\varnothing\}$, int $A \subseteq \psi(A)$ and $\psi(\varnothing)=\varnothing$.

The set of all operations on a space $X$ will be denoted by $\mathcal{O}(X)$.

Observation 2.2. It is easy to check that some examples of operations on a space $X$ are the well known operators viz. int, intcl, intcl $\delta$, clint, intclint, clintcl.

Definition 2.3([6]). Let $\psi$ denote an operation on a space $(X, \tau)$. Then a subset $A$ of $X$ is called $\psi$-open if $A \subseteq \psi(A)$. Complement of $\psi$-open sets will be called $\psi$-closed sets. The family of all $\psi$-open (resp. $\psi$-closed) subsets of $X$ is denoted by $\psi \mathcal{O}(X)($ resp. $\psi \mathrm{C}(X))$.

Observation 2.4. It is clear that if $\psi$ stands for any of the operators int, intcl, intcl $_{\delta}$, clint, intclint, clintcl, then $\psi$-openness of a subset $A$ of $X$ coincides with the openness, preopenness, $\delta$-preopenness, semi-openness, $\alpha$-openness and $\beta$-openness of $A$ (see $[7,16,18,20,10,1])$ respectively.

Definition 2.5([6]). Let $(X, \tau)$ be a topological space, $\psi \in \mathcal{O}(X)$ and $A \subseteq X$. Then the intersection of all $\psi$-closed sets containing $A$ is called the $\psi$-closure of $A$, denoted by $\psi$-cl $A$; alternately, $\psi$-cl $A$ is the smallest $\psi$-closed set containing $A$.

It is known [11] that $x \in \psi$-cl $A$ iff $A \cap U \neq \varnothing$, for all $U$ with $x \in U \in \psi \mathcal{O}(X)$.

Observation 2.6. Obviously if one takes interior as the operation $\psi$, then $\psi$-closure becomes equivalent to the usual closure. Similarly, $\psi$-closure becomes $p c l, p c l_{\delta}, s c l$, $\alpha-c l, \beta-c l$, if $\psi$ is taken to stand for the operators intcl, intcl $\delta$, clint, intclint and clintcl respectively (see $[7,16,18,20,10,1]$ for details).

Definition 2.7. Let $\psi$ be an operation on a topological space $(X, \tau)$ and $A \subseteq X$. Then the subsets $\bigwedge_{\psi}(A)$ and $\bigvee_{\psi}(A)$ are defined as follows :

$\bigwedge_{\psi}(A)=\cap\{G: A \subseteq G, G \in \psi \mathcal{O}(X)\}$ and $\bigvee_{\psi}(A)=\cup\left\{H: H \subseteq A, H^{c} \in \psi \mathcal{O}(X)\right\}$.

Remark 2.8. In a topological space $X$ if we take $\psi=$ int (resp. intcl, clint, intclint, clintcl) then the concept of $\bigwedge_{\psi}(A)$ coincides with that of $A^{\wedge}$ [15] (resp. $\left.\bigwedge_{p}(A)[9], A \wedge_{s}[3], \bigwedge_{\alpha}(A)[4], \bigwedge s p(A)[17]\right)$.

Proposition 2.9. Let $A, B$ and $\left\{A_{\alpha}: \alpha \in \Delta\right\}$ be subsets of a topological space 
$(X, \tau)$ and $\psi \in \mathcal{O}(X)$. Then the following properties hold:
(a) $A \subseteq \bigwedge_{\psi}(A)$;
(b) If $A \subseteq B$, then $\bigwedge_{\psi}(A) \subseteq \bigwedge_{\psi}(B)$;
(c) $\bigwedge_{\psi}\left(\bigwedge_{\psi}(A)\right)=\bigwedge_{\psi}(A)$;
(d) $\bigwedge_{\psi}\left[\bigcup_{\alpha \in \Delta} A_{\alpha}\right]=\bigcup_{\alpha \in \Delta}\left[\bigwedge_{\psi}\left(A_{\alpha}\right)\right]$
(e) If $A \in \psi \mathcal{O}(X)$, then $A=\bigwedge_{\psi}(A)$;
(f) $\bigwedge_{\psi}\left(A^{c}\right)=\left(\bigvee_{\psi}(A)\right)^{c}$;
(g) $\bigvee_{\psi}(A) \subseteq A$;
(h) If $A^{c} \in \psi \mathcal{O}(X)$, then $\bigvee_{\psi}(A)=A$;
(i) $A \subseteq B$, then $\bigvee_{\psi}(A) \subseteq \bigvee_{\psi}(B)$

Proof. Straight forward.

Example 2.10. Let $X=\{a, b, c\}$ and $\tau=\{\varnothing,\{a\},\{b\},\{a, c\},\{a, b\}, X\}$. Then $(X, \tau)$ is a topological space. Consider the map $\psi: \mathcal{P}(X) \rightarrow \mathcal{P}(X)$ defined by $\psi(\{a\})=\{a, c\} ; \psi(\{b\})=\{b\} ; \psi(\{c\})=\varnothing ; \psi(\{a, b\})=X ; \psi(\{a, c\})=\{a, c\} ;$ $\psi(\{b, c\})=\{b\} ; \psi(X)=X$ and $\psi(\{\varnothing\})=\varnothing$. Then $\psi$ is an operation on $(X, \tau)$. But it is easy to verify that $\bigwedge_{\psi}(\{b\})=\{b\} ; \bigwedge_{\psi}(\{b, c\})=X ; \bigwedge_{\psi}(\{a, b\})=\{a, b\}$. Thus $\bigwedge_{\psi}(\{b, c\}) \cap \bigwedge_{\psi}(\{a, b\}) \neq \bigwedge_{\psi}(\{b\})$.

Definition 2.11. Let $\psi$ be an operation on a topological space $(X, \tau)$. Then a subset $A$ is said to be a $\bigwedge_{\psi}$-set (resp. $\bigvee_{\psi}$-set) if $A=\bigwedge_{\psi}(A)\left(\right.$ resp. $\left.A=\bigvee_{\psi}(A)\right)$.

Remark 2.12. In the above definition if we take $\psi=$ int (resp. intcl, clint, intcl ${ }_{\delta}$, intclint, clintcl) then a $\bigwedge_{\psi}$-set reduces to a $\bigwedge_{\text {-set }}$ [15] (resp. $\bigwedge_{p}$-set [9], $\bigwedge_{s}$-set [3], $\bigwedge_{\alpha}$-set [4], $\bigwedge s p$-set [17]).

Theorem 2.13. Let $\psi$ be an operation on a topological space $(X, \tau)$. Then

(a) $\varnothing, X$ are $\Lambda_{\psi}$-sets (resp. $\varnothing, X$ are $\bigvee_{\psi}$-sets).

(b) The union of $\bigwedge_{\psi}$-sets (resp. $\bigvee_{\psi}$-sets) is a $\bigwedge_{\psi}$-set (resp. $\bigvee_{\psi}$-set).

(c) The intersection of $\bigwedge_{\psi}$-sets (resp. $\bigvee_{\psi}$-sets) is a $\bigwedge_{\psi}$-set (resp. $\bigvee_{\psi}$-set).

Proof. (a) Follows from Definition 2.7.

(b) Let $\left\{B_{\alpha}: \alpha \in \Omega\right\}$ be a family of $\bigwedge_{\psi}$-sets in a topological space $(X, \tau)$. Then by Proposition 2.9(d) and Definition 2.11, $\bigcup_{\alpha \in \Omega} B_{\alpha}=\bigcup_{\alpha \in \Omega}\left[\bigwedge_{\psi}\left(B_{\alpha}\right)\right]=\bigwedge_{\psi}\left[\bigcup_{\alpha \in \Omega} B_{\alpha}\right]$.

(c) Let $\left\{B_{\alpha}: \alpha \in \Omega\right\}$ be a family of $\bigwedge_{\psi}$-sets in a topological space $(X, \tau)$. Then by Proposition 2.9(b) and Definition 2.11, $\bigwedge_{\psi}\left[\bigcap_{\alpha \in \Omega} B_{\alpha}\right] \subseteq \bigcap_{\alpha \in \Omega}\left[\bigwedge_{\psi}\left(B_{\alpha}\right)\right]=\bigcap_{\alpha \in \Omega} B_{\alpha}$. Hence by Proposition 2.9(a), $\bigcap_{\alpha \in \Omega} B_{\alpha}=\bigwedge_{\psi}\left[\bigcap_{\alpha \in \Omega} B_{\alpha \in \Omega}\right]$.

Remark 2.14. If $\psi$ be an operation on a topological space $X$, then the family of $\Lambda_{\psi}$-sets (resp. $\bigvee_{\psi}$-sets) of $X$ forms a topology on $X$, containing the class of all $\psi$-open (resp. $\psi$-closed) sets. Thus from Theorem 2.13, it follows that $\left(X, \bigwedge_{\psi}\right)$ and $\left(X, \bigvee_{\psi}\right)$ are Alexandroff spaces [2] i.e., arbitrary intersection of open sets of $X$ is open in $X$. 
Definition 2.15. Let $\psi$ be an operation on a topological space $(X, \tau)$. Then the space $X$ is said to be a

(i) $\psi$ - $T_{0}$ space [11] if for any pair of distinct points in $X$ there exists a $\psi$-open set containing one of the points but not the other.

(ii) $\psi-T_{1}$ space [11] if for any two distinct points $x, y$ of $X$, there exist $\psi$-open sets $U, V$ such that $x \in U, y \notin U, x \notin V, y \in V$.

Theorem 2.16. Let $\psi$ be an operation on a topological space $(X, \tau)$. Then the following are equivalent :

(a) $(X, \tau)$ is $\psi-T_{1}$;

(b) Every subset of $X$ is a $\bigwedge_{\psi}$-set;

(c) Every subset of $X$ is a $\bigvee_{\psi}$-set.

Proof. It is obvious that (b) $\Leftrightarrow$ (c).

(a) $\Rightarrow$ (c) : Let $A$ be any subset of $X$. Since $A=\cup\{\{x\}: x \in A\}, A$ is the union of $\psi$-closed sets (as $X$ is a $\psi$-T $T_{1}$ space, see [19] for details), hence $A$ is a $\bigvee_{\psi}$-set.

(b) $\Rightarrow$ (a) : Let $x \in X$. Then by (b), $\{x\}$ is a $\bigwedge_{\psi}$-set. Let $x, y$ be any two distinct points of $X$. Then $y \notin \bigwedge_{\psi}(\{x\})=\{x\}$. So by definition of $\bigwedge_{\psi}$-sets, there exists $U \in \psi \mathcal{O}(X)$ such that $x \in U$ but $y \notin U$. Similarly the other case can be done. Thus $(X, \tau)$ is $\psi-T_{1}$.

\section{Generalized $\bigwedge_{\psi}$-set}

Definition 3.1. Let $\psi$ be an operation on a topological space $(X, \tau)$. Then a subset $A$ of $X$ is said to be a $g . \bigwedge_{\psi}$-set if $\bigwedge_{\psi}(A) \subseteq F$ whenever $A \subseteq F$ and $F$ is $\psi$-closed. The complement of a $g \cdot \bigwedge_{\psi}$-set is known as a $g \bigvee_{\psi}$-set.

Observation 3.2. It is easy to check that the above definition of a $g \bigwedge_{\psi}$-set unifies the existing definitions of $g \cdot \wedge$-set [15],g. $\bigwedge_{p}$-set [9],g. $\bigwedge_{s}$-set [3], $g \cdot \bigwedge_{\alpha}{ }^{-s e t}[4]$ and $g . \wedge s p$-set [17] if the operators int, intcl, clint, intclint, clintcl respectively take the role of $\psi$. Similarly we can obtain $g$. $\bigvee$-set [15] ( resp. $g . \bigvee_{p}$-set $[9], g . \bigvee_{s}$-set [3], $g . \bigvee_{\alpha}$-set [4]) by replacing $\psi$ by int (resp. intcl, clint, intclint).

By $D^{\wedge_{\psi}}$ (resp. $D^{\bigvee_{\psi}}$ ) we shall denote the family of all $g \cdot \wedge_{\psi^{-}}$sets (resp. $g \cdot \bigvee_{\psi^{-}}$ sets) of a topological space $(X, \tau)$ with the operation $\psi$.

Theorem 3.3. Let $\psi$ be an operation on a space $X$.

(a) Every $\bigwedge_{\psi}$-set is a $g . \bigwedge_{\psi}$-set;

(b) Every $\bigvee_{\psi}$-set is a $g . \bigvee_{\psi}$-set;

(c) $D^{\wedge_{\psi}}$ (resp. $D^{\vee_{\psi}}$ ) is closed under arbitrary union (resp. intersection).

Proof. (a) Follows from Definition 3.1.

(b) Let $A$ be a $\bigvee_{\psi}$-set. Then $A=\bigvee_{\psi}(A)$. By Proposition 2.9(f), $\bigwedge_{\psi}\left(A^{c}\right)=$ $\left(\bigvee_{\psi}(A)\right)^{c}=A^{c}$. Thus by (a) and Definition 3.1, $A$ is a $g \cdot \bigvee_{\psi}$-set.

(c) Let $\left\{A_{\alpha}: \alpha \in \Delta\right\}$ be a family of members of $D^{\wedge}$. Then by Proposition $2.9(\mathrm{~d}), \bigwedge_{\psi}\left[\bigcup_{\alpha \in \Delta} A_{\alpha}\right]=\bigcup_{\alpha \in \Delta}\left[\bigwedge_{\psi}\left(A_{\alpha}\right)\right]$. Hence by hypothesis and Definition 3.1, 
$\bigcup_{\alpha \in \Delta} A_{\alpha} \in D^{\wedge_{\psi}}$

The proof of the next part can be shown in a similar way.

Example 3.4. Let $X=\{a, b, c\}$ and $\tau=\{\varnothing,\{a, c\}, X\}$. Then $(X, \tau)$ is a topological space. Consider the mapping $\psi: \mathcal{P}(X) \rightarrow \mathcal{P}(X)$ defined by $\psi(A)=i n t A$. Then $\psi$ is an operation on the topological space $(X, \tau)$. We note that $\{b\}$ is a $g \cdot \bigwedge_{\psi^{-}}$-set which is not a $\bigwedge_{\psi}$-set.

Theorem 3.5. Let $\psi$ be an operation on a topological space $X$. For each $x \in X$,

(a) $\{x\}$ is $\psi$-open or $\{x\}^{c}$ is a $g . \bigwedge_{\psi}$-set.

(b) $\{x\}$ is $\psi$-open or $\{x\}$ is a $g . \bigvee_{\psi}^{\text {-set. }}$

Proof. (a) Suppose that $\{x\}$ is not a $\psi$-open set. Then the only $\psi$-closed set $F$ containing $\{x\}^{c}$ is $X$. Thus $\bigwedge_{\psi}\left(\{x\}^{c}\right) \subseteq F=X$ and thus $\{x\}^{c}$ is a $g \cdot \bigwedge_{\psi^{-}}$set of $X$. (b) Follows from (a) and Definition 3.1.

Proposition 3.6. Let $\psi$ be an operation on a space $X$. If $A$ is a $g . \Lambda_{\psi}$-set and $A \subseteq B \subseteq \bigwedge_{\psi}(A)$, then $B$ is a $g . \bigwedge_{\psi}$-set of $(X, \tau)$.

Proof. Since $A \subseteq B \subseteq \bigwedge_{\psi}(A)$, by (b) and (c) of Proposition 2.9, we have $\bigwedge_{\psi}(A)=$ $\bigwedge_{\psi}(B)$. Let $F$ be any $\psi$-closed subset of $X$ such that $B \subseteq F$. Then, $\bigwedge_{\psi}(B)=$ $\bigwedge_{\psi}(A) \subseteq F$ since $A \subseteq B$ and $A$ is a $g \cdot \bigwedge_{\psi}$-set.

Theorem 3.7. Let $\psi$ be an operation on a topological space $(X, \tau)$. A subset $A$ is a $g . \bigvee_{\psi}$-set iff $U \subseteq \bigvee_{\psi}(A)$ whenever $U \subseteq A$ and $U \in \psi \mathcal{O}(X)$.

Proof. Let $U$ be a $\psi$-open set such that $U \subseteq A$. Then $U^{c}$ is $\psi$-closed and $A^{c} \subseteq U^{c}$, we have by Definition 3.1, $\bigwedge_{\psi}\left(A^{c}\right) \subseteq U^{c}$. Hence by Proposition 2.9(f), $\left(\bigvee_{\psi}(A)\right)^{c} \subseteq U^{c}$. Thus $U \subseteq \bigvee_{\psi}(A)$.

Conversely, let $F$ be a $\psi$-closed subset of $X$ such that $A^{c} \subseteq F$. Since $F^{c}$ is $\psi$-open and $F^{c} \subseteq A$, by hypothesis we have $F^{c} \subseteq \bigvee_{\psi}(A)$. Then by Proposition $2.9(\mathrm{f}), \bigwedge_{\psi}\left(A^{c}\right)=\left(\bigvee_{\psi}(A)\right)^{c} \subseteq F$. Thus $A^{c}$ is a $g \cdot \bigwedge_{\psi}$-set i.e., $A$ is a $g . \bigvee_{\psi}$-set.

Corollary 3.8. Let $\psi$ be an operation on a space $X$ and $A$ be $a g . \bigvee_{\psi}$-set. Then for every $\psi$-closed set $F$ such that $\bigvee_{\psi}(A) \cup A^{c} \subseteq F, F=X$ holds.

Proof. The assumption $\bigvee_{\psi}(A) \cup A^{c} \subseteq F$ implies that $F^{c} \subseteq\left(\bigvee_{\psi}(A)\right)^{c} \cap A$. Since $A$ is a $g . \bigvee_{\psi^{-s e t}}$, then by Theorem 3.7, we have $\bigvee_{\psi}(A) \supseteq F^{c}$. On the other hand $\left(\bigvee_{\psi}(A)\right)^{c} \supseteq\left(\bigvee_{\psi}(A)\right)^{c} \cap A \supseteq F^{c}$ and $\varnothing=\bigvee_{\psi}(A) \cap\left(\bigvee_{\psi}(A)\right)^{c} \supseteq F^{c}$. Therefore, we have $F=X$.

Corollary 3.9. Let $\psi$ be an operation on a topological space $(X, \tau)$. If $A$ is a $g . \bigvee_{\psi}$-set then $\bigvee_{\psi}(A) \cup A^{c}$ is a $\psi$-closed set if and only if $A$ is a $\bigvee_{\psi}$-set.

Proof. Since $\bigvee_{\psi}(A)=A, \bigvee_{\psi}(A) \cup A^{c}=X$ is $\psi$-closed.

Conversely, by Corollary 3.8, $\bigvee_{\psi}(A) \cup A^{c}=X$. Thus $\left(\bigvee_{\psi}(A)\right)^{c} \cap A=\varnothing$. Hence by Proposition $2.9(\mathrm{~g}), \bigvee_{\psi}(A)=A$. 


\section{Comparison of $(X, \tau)$ and the space $\left(X, \bigwedge_{\psi}\right)$}

Definition 4.1. Let $\psi$ be an operation on a topological space $(X, \tau)$. A subset $A$ of $X$ is said to be $\psi g$-closed if $\psi$-cl $(A) \subseteq U$ holds whenever $A \subseteq U$ and $U \in \psi \mathcal{O}(X)$. The complement of a $\psi g$-closed set is called a $\psi g$-open set.

Definition 4.2. Let $\psi$ be an operation on a topological space $(X, \tau)$. Then $(X, \tau)$ is said to be a $\psi-T_{1 / 2}$ space if every $\psi g$-closed set in $(X, \tau)$ is $\psi$-closed.

Theorem 4.3. Let $\psi$ be an operation on a topological space $(X, \tau)$. Then the following are equivalent :

(a) $(X, \tau)$ is a $\psi-T_{1 / 2}$ space;

(b) For each $x \in X,\{x\}$ is either $\psi$-open or $\psi$-closed;

(c) Every $g . \bigvee_{\psi}$-set is a $\bigvee_{\psi}$-set.

Proof. (a) $\Rightarrow$ (b): Let $x$ be a point of $X$. Then $\{x\}$ is either $\psi$-closed or not $\psi$-closed. If $\{x\}$ is $\psi$-closed then we have nothing to show. Suppose now that $\{x\}$ is not $\psi$-closed. Then $\{x\}^{c}$ is not $\psi$-open and hence $\{x\}^{c} \subseteq X \Rightarrow \psi-c l\left(\{x\}^{c}\right) \subseteq X$, i.e., $\{x\}^{c}$ is $\psi g$-closed. Since $(X, \tau)$ is a $\psi$ - $T_{1 / 2}$ space, $\{x\}^{c}$ is $\psi$-closed and hence $\{x\}$ is $\psi$-open.

(b) $\Rightarrow$ (a): Suppose that $A$ be a $\psi g$-closed subset of $(X, \tau)$. We shall show that $\psi-\operatorname{cl}(A) \subseteq A$. Let $x \in \psi$-cl $(A)$. Then by (b), $\{x\}$ is either $\psi$-open or $\psi$-closed. If $\{x\}$ is $\psi$-open then $x \in A$ (as $x \in \psi$-cl $(A)$ ). If $\{x\}$ is $\psi$-closed, then as $\psi$-cl $(A) \backslash A$ does not contain any non-empty $\psi$-closed set (in fact, if any $\psi$-closed set $F$ is contained in $\psi$-cl $(A) \backslash A$ then $A \subseteq F^{c}$ and hence by $\psi g$-closedness of $A, \psi$-cl $(A) \subseteq F^{c}$. Thus $F \subseteq \psi-c l(A) \cap(\psi-c l(A))^{c}=\varnothing$ i.e., $\left.F=\varnothing\right)$, we have $x \in A($ as $x \notin \psi-c l(A) \backslash A)$. Thus in both the cases $x \in A$.

(a) $\Rightarrow$ (c): Suppose that there exists a $g \cdot \bigvee_{\psi}$-set $B$ which is not a $\bigvee_{\psi}$-set. Then $\bigvee_{\psi}(B) \varsubsetneqq B$. Thus there exists $x \in B$ such that $x \notin \bigvee_{\psi}(B)$. We shall now show that $\{x\}$ is neither a $\psi$-open nor a $\psi$-closed set which contradicts (b). Suppose that $\{x\}$ is $\psi$-closed. Then $\{x\} \subseteq B \Rightarrow B^{c} \subseteq\{x\}^{c}$. So by Proposition 2.9(b) and (e), $\bigwedge_{\psi}\left(B^{c}\right) \subseteq \bigwedge_{\psi}\left(\{x\}^{c}\right)=\{x\}^{c}$. Thus by Proposition 2.9(f), $\left(\bigvee_{\psi}(B)\right)^{c} \subseteq\{x\}^{c}$ i.e., $\{x\} \subseteq \bigvee_{\psi}(B)$ - a contradiction. Next, let $\{x\}$ be $\psi$-open. Since $\{x\} \subseteq B$ and $B$ is a $g . \bigvee_{\psi}$-set, $\{x\} \subseteq \bigvee_{\psi}(B)$ (by Theorem 3.7) - a contradiction.

(c) $\Rightarrow\left(\right.$ a): Suppose that $(X, \tau)$ is not a $\psi-T_{1 / 2}$ space. Then there exists a $\psi g$-closed set $B$ which is not $\psi$-closed. Since $B$ is not $\psi$-closed, there exists a point $x$ such that $x \notin B$ and $x \in \psi-c l(B)$. Then by Theorem 3.5, $\{x\}$ is a $\psi$-open set or a $g . \bigvee_{\psi^{-s e t}}$. Case $-1:\{x\}$ is a $\psi$-open set: Then as $x \in \psi$-cl $(B)$, we have $\{x\} \cap B \neq \varnothing$, i.e., $x \in B$. This is a contradiction.

Case $-2:\{x\}$ is a $g . \bigvee_{\psi}$-set : Then $\{x\}$ is either $\psi$-closed or not $\psi$-closed. If $\{x\}$ is not $\psi$-closed, we have $\bigvee_{\psi}(\{x\})=\varnothing$. Hence $\{x\}$ is not a $\bigvee_{\psi}$-set. This is a contradiction to (b).

If $\{x\}$ is $\psi$-closed, then $B \subseteq\{x\}^{c}$ (as $x \notin B$ ). So $\psi$-cl $(B) \subseteq\{x\}^{c}$ (as $B$ is $\psi$ g-closed and $\{x\}^{c}$ is $\psi$-open). Thus $\{x\} \subseteq(\psi-c l(B))^{c}$. This contradicts the fact that $x \in \psi$ $\operatorname{cl}(B)$. 
Definition 4.4. A topological space $(X, \tau)$ is said to be $\psi-R_{0}[11,19]$ if for each $\psi$-open set $U$ and each $x \in U, \psi-c l(\{x\}) \subseteq U$.

Theorem 4.5. A topological space $(X, \tau)$ with an operation $\psi$ is $\psi$ - $R_{0}$ iff the topological space $\left(X, \bigwedge_{\psi}\right)$ is $R_{0}$.

Proof. Let $(X, \tau)$ be $\psi-R_{0}$. Let $V \in \bigwedge_{\psi}$ and $x \in V$. Then $x \in V=\bigwedge_{\psi}(V)=$ $\cap\{U: V \subseteq U ; U \in \psi \mathcal{O}(X)\}$ and so $x \in U$ for each $U \in \psi \mathcal{O}(X)$ containing $V$. Since $(X, \tau)$ is $\psi-R_{0}, \psi-c l(\{x\}) \subseteq U$ for each $U \in \psi \mathcal{O}(X)$ containing $V$. Hence $\psi-c l(\{x\}) \subseteq \cap\{U: V \subseteq U ; U \in \bar{\psi} \mathcal{O}(X)\}=\bigwedge_{\psi}(V)=V$. Since $\psi \mathcal{O}(X) \subseteq \bigwedge_{\psi}$, we have $\bigwedge_{\psi}-c l(\{x\}) \subseteq \psi-c l(\{x\}) \subseteq V$. Thus $\left(X, \bigwedge_{\psi}\right)$ is $R_{0}$.

Conversely, suppose that $\left(X, \bigwedge_{\psi}\right)$ is $R_{0}$. Let $V \in \psi \mathcal{O}(X)$ and $x \in V$. Since $\psi \mathcal{O}(X) \subseteq \bigwedge_{\psi}$, we have $\bigwedge_{\psi}-c l(\{x\}) \subseteq V$. Since $X \backslash \bigwedge_{\psi}-c l(\{x\}) \in \bigwedge_{\psi}$, we have $X \backslash \bigwedge_{\psi}-c l(\{x\})=\cap\left\{U: X \backslash \bigwedge_{\psi}-c l(\{x\}) \subseteq U ; U \in \psi \mathcal{O}(X)\right\}$. So there exists $U \in \psi \mathcal{O}(X)$ such that $X \backslash \bigwedge_{\psi}-c l(\{x\}) \subseteq U$ and $x \notin U$ and hence $x \in X \backslash U \subseteq \bigwedge_{\psi^{-}}$ $\operatorname{cl}(\{x\})$. Since $X \backslash U$ is $\psi$-closed, $\psi$-cl $(\{x\}) \subseteq X \backslash U \subseteq V$. This shows that $(X, \tau)$ is $\psi-R_{0}$.

Theorem 4.6. A topological space $(X, \tau)$ with an operation $\psi$ is $\psi-T_{0}$ iff the topological space $\left(X, \bigwedge_{\psi}\right)$ is $T_{0}$.

Proof. One part of the proof is trivial as $\psi \mathcal{O}(X) \subseteq \bigwedge_{\psi}$.

Conversely, let $x$ and $y$ be any pair of distinct points of $X$. Since $\left(X, \bigwedge_{\psi}\right)$ is $\bigwedge_{\psi}-T_{0}$, without loss of generality we may assume that there exists $V \in \bigwedge_{\psi}$ such that $x \in V$ and $y \notin V$. Then there exists $U \in \psi \mathcal{O}(X)$ such that $V \subseteq U$ and $y \notin U$. Hence $x \in U$ and $y \notin U$. Thus $(X, \tau)$ is $\psi-T_{0}$.

Theorem 4.7. A topological space $(X, \tau)$ with an operation $\psi$ is $\psi-T_{1}$ iff the topological space $\left(X, \bigwedge_{\psi}\right)$ is discrete.

Proof. In a $\psi$ - $T_{1}$-space, by Theorem 2.16, for each $x \in X,\{x\}$ is a $\bigwedge_{\psi}$-set. So for every $x \in X,\{x\}$ is open in $\left(X, \bigwedge_{\psi}\right)$. Hence $\left(X, \bigwedge_{\psi}\right)$ is a discrete space.

Conversely, suppose that $\left(X, \bigwedge_{\psi}\right)$ is a discrete space and $x, y$ are two distinct points of $X$. Then $\{x\}$ and $\{y\}$ are open in $\left(X, \Lambda_{\psi}\right)$. Thus $\{x\}$ and $\{y\}$ are $\bigwedge_{\psi}$-sets. So $y \notin \bigwedge_{\psi}(\{x\})$ and $x \notin \bigwedge_{\psi}(\{y\})$. Thus there exist $\psi$-open sets $G$ and $H$ such that $y \notin G$, but $x \in G$ and $x \notin H$, but $y \in H$. Thus $(X, \tau)$ is a $\psi$ - $T_{1}$-space.

\section{References}

[1] M. E. Abd El-Monsef, S. N. El-Deeb and R. A. Mahmoud, $\beta$-open sets and $\beta$ continuous mappings, Bull. Fac. Sci. Assiut Univ., 12(1983), 77-90.

[2] P. Alexandroff, Diskrete Räume, Math. Sb., 2(1937), 501-508.

[3] M. Caldas and J. Dontchev, G. $\bigwedge_{s}$-sets and $G . \bigvee_{s}-$ sets, Mem. Fac. Sci. Kochi Univ. (Math.), 21(2000), 21-30. 
[4] M. Caldas, D. N. Georgiou and S. Jafari, Study of $(\bigwedge, \alpha)$-closed sets and related notions in topological spaces, Bull. Malays. Math. Sci. Soc., 30(2)(1)(2007), 23-36.

[5] M. Caldas, S. Jafari and T. Noiri, On $\bigwedge_{b}$ sets and the associated topology $\tau^{\wedge_{b}}$, Acta Math. Hungar., 110(2006), 337-345.

[6] Á. Császár, Generalized open sets, Acta. Math. Hungar., 75(1-2)(1997), 65-87.

[7] J. Dugundji, Topology, Allyn and Bacon, Boston, 1966.

[8] E. Ekici and B. Roy, New generalized topologies on generalized topological spaces due to Császár, Acta Math. Hungar., 132(2011), 117-124.

[9] M. Ganster, S. Jafari and T. Noiri, On pre- $\bigwedge$-sets and pre- $\bigvee$-sets, Acta Math. Hungar., 95(2002), 337-343.

[10] S. Jafari, Rare $\alpha$-continuity, Bull. Malays. Math. Sci. Soc., 28(2)(2005), 157-161.

[11] M. Kücük and $\dot{I}$. Zorlutuna, A unified theory for weak separation properties, Internat. J. Math and Math. Sci., 24(11)(2000), 765-772.

[12] M. Kücük and $\dot{I}$. Zorlutuna, A unification on compactness and closedness, Soochow J. Math., 29(3)(2003), 221-233.

[13] N. Levine, Semi-open sets and semi-continuity in topological spaces, Amer. Math. Monthly, 70(1963), 36-41.

[14] R. A. Mahmoud and M. E. Abd El-Monsef, $\beta$-irresolute and $\beta$-topological invariant, Proc. Pakistan Acad. Sci., 27(3)(1990), 285-296.

[15] H. Maki, Generalized $\bigwedge$-sets and the associated closure operator, Special issue in commemoration of Prof. Kazusada IKEDA's retirement, (1986), 139-146.

[16] A. S. Mashhour, M. E. Abd El-Monsef and S. N. El-Deeb, On precontinuous and weak precontinuous mappings, Proc. Math. Phys. Soc. Egypt, 53(1982), 47-53.

[17] T. Noiri and E. Hatir, $\Lambda$ sp-sets and some weak separation axioms, Acta. Math. Hungar., 103(3)(2004), 225-232.

[18] S. Raychaudhuri and M. N. Mukherjee, On $\delta$-almost continuity and $\delta$-preopen sets, Bull. Inst. Math. Acad. Sinica, 21(1993), 357-366.

[19] B. Roy and M. N. Mukherjee, A unified theory for $R_{0}, R_{1}$ and certain other separation properties and their variant forms, Bol. Soc. Paran. Mat., 28(2)(2010), 9-18.

[20] I. L. Reilly and M. K. Vamanamurthy, On semi compact spaces, Bull. Malays. Math. Soc., $7(2)(1984), 61-67$. 